Aspekten der Selbstbestimmung und des Persönlichkeitsschutzes adäquat zu thematisieren. Dafür entwickelt sie Qualitätsstandards und plädiert dafür, nicht nur die Veröffentlichung des Privaten als journalistische Leistung anzuerkennen, sondern diese an journalistischen Qualitätskriterien zu messen.

Mit dieser Studie liefert Herrmann nicht nur einen wichtigen Beitrag zur Talkshow-Diskussion in unserem Fach, sondern auch neue Ansatzpunkte $\mathrm{zu}$ einer produktiven Weiterführung der feministischen Theoriediskussion zur Verschränkung von Öffentlichkeit und Privatheit.

\section{Johanna Dorer}

\section{Nicola Döring \\ Sozialpsychologie des Internet}

Die Bedeutung des Internet für Kommunikationsprozesse, Identitäten, soziale Beziehungen und Gruppen

2. vollständig überarbeitete und erweiterte Auflage

Göttingen u. a.: Hogrefe Verlag für Psychologie, 2003. $-662 \mathrm{~S}$.

\section{ISBN 3-8017-1466-7}

Auch wenn erst 4 Jahre seit ihrem Erscheinen vergangen sind, kann man die erste Auflage von Nicola Dörings „Sozialpsychologie des Internet" getrost als Klassiker der Internetforschung bezeichnen. Es ist ein Fachbuch, ein Lehrbuch und ein Nachschlagewerk, das in zahlreichen Rezensionen positiv aufgenommen wurde (z. B. Zeitschrift für Experimentelle Psychologie, 2000, 48, S. 74-75) und auch fachübergreifend viel Beachtung fand. Wie sieht es nun mit der zweiten Auflage dieses nunmehr über 600 Seiten starken Buches aus, das, so ist im Vorwort zu lesen, vollständig überarbeitet und erweitert wurde? Wie reagiert die Autorin auf die aktuelle Entwicklung des Internets, arbeitet sie die neue Forschungsliteratur auf, kommen neue Aspekte hinzu? Im Folgenden werden diese Fragen im Mittelpunkt stehen, doch sollen nicht nur die Unterschiede zur Erstauflage in den Blick genommen werden. Ziel ist auch, im sukzessiven Durchgang durch die einzelnen Kapitel über das gesamte Werk zu informieren und es kritisch zu würdigen.

Das Buch besteht aus neun Kapiteln, einem Serviceteil, einer umfassenden Literaturliste und einem Index. Es beginnt mit allgemeinen Erläuterungen zur Entwicklung und Bedeutung des Internets (Kap. 1). Hier werden Informationen zum technischen Hintergrund, zur Diffusion und zur Nutzung des Internets, zu juristischen Fragen und zum Stellenwert des Internets in unserer Gesellschaft gegeben. Die Ausführungen in diesem Kapitel wurden gegenüber der Erstauflage zu Recht komprimiert, denn viele dieser Informationen sind mittlerweile bekannt bzw. in jedem Einführungsbuch nachzulesen. Auch das zweite Kapitel, in dem zahlreiche Internetanwendungen vorgestellt werden, hat Einführungscharakter. Positiv hervorzuheben ist hier zum einen, dass Döring auch auf solche Internetangebote eingeht, die dem Leser/der Leserin nicht so bekannt sein dürften (z. B. Multi User Domains), zum andern, dass sie zeigt, wie die herkömmliche Individual- und Massenkommunikation in das Internet eingebunden wird (z. B. Online-Zeitungen). Demgegenüber hätten die Ausführungen zu den mittlerweile vielfach genutzten Internetdiensten (E-Mail, Chat) etwas kürzer ausfallen können.

Kapitel 3 führt weg von der medienkundlichen Einführung zur Diskussion verschiedener Forschungsansätze. Döring stellt hier insgesamt elf Theorien zur computervermittelten Kommunikation $(\mathrm{CvK})$ vor, die sie in drei Blöcke teilt (Theorien zur Medienwahl, Theorien zu Medienmerkmalen, Theorien zum medialen Kommunikationsverhalten), jeweils kritisch kommentiert und in ein medienökologisches Rahmenmodell integriert. Dieses medienübergreifende Modell soll dazu beitragen, die Einseitigkeit einer technikdeterministischen bzw. kulturalistischen Sichtweise zu vermeiden. Es stellt mit den fünf Komponenten a) Medienwahl, b) Medienmerkmale, c) mediales Kommunikationsverhalten, d) kurzfristige soziale Effekte, e) langfristige soziale Folgen in der Tat eine gelungene Verbindung von medien- und nutzerzentrierten Ansätzen dar und trägt damit den Wechselwirkungen zwischen Mensch und Medienumwelt Rechnung. Kap. 4 leitet von theoretischen Aspekten zu empirisch-methodischen Fragen über. Ausführlicher als in der Erstauflage werden Forschungsdesigns (z. B. grundlagenwissenschaftliche Untersuchungen), Auswahlverfahren (z. B. Vollerhebungen), Datenerhebungstechniken (z. B. Log-File-Analysen) und Datenauswertungsmethoden (z. B. Online-Data-Mining) vorge- 
stellt und exemplarisch auf konkrete Studien zur Internetforschung bezogen. Auch ethische Richtlinien, die für Forschungsaktivitäten im Netz gelten sollten, werden erörtert. Eben weil, wie Döring schreibt, die „prinzipielle Zugänglichkeit von Online-Dokumenten [...] nicht gleichzusetzen mit ihrer intentionalen Publikation in einer größeren Öffentlichkeit als dem Kreis der aktuell Beteiligten" (S. 238) ist, sind solche Überlegungen von großer Bedeutung.

Kap. 5 gibt einen Überblick über aktuelle Themen der sozialpsychologischen OnlineForschung und wendet sich damit dem Schwerpunkt der Arbeit zu. Döring behandelt hier sowohl grundlagenwissenschaftliche Fragen (z. B. Wirklichkeitskonstruktionen und soziale Verhaltensweisen im Internet) als auch anwendungsbezogene Aspekte (z. B. Auswirkungen der Internet-Nutzung auf Gesundheit und Umwelt, auf Erziehung, Bildung und Forschung, Wirtschaft, Arbeit, Politik und Kultur). In den folgenden drei Kapiteln stehen wichtige sozialpsychologische Konstrukte im Mittelpunkt: Identität (Kap. 6), soziale Beziehung (Kap. 7) und Gruppe (Kap. 8). Kap. 6 zeigt, welche Unterschiede zwischen Offlineund Online-Identitäten bestehen, Kap. 7, wie sich soziale Beziehungen im Netz gestalten. Die behandelten Aspekte reichen vom Geschlechterwechsel bis zur Online-Sucht, von Online-Tagebüchern bis zu Beziehungskrisen im Internet. Außerdem nimmt Döring hier kritisch Stellung zu der weit verbreiteten Annahme, durch Online-Kontakte komme es zu einer Verarmung bestehender sozialer Beziehungen und zu einer Flucht in virtuelle Scheinbeziehungen. Eine differenzierte Sicht auf die Internetnutzung findet sich auch in Kap. 8, wo argumentiert wird, dass die Mitgliedschaft in Online-Gruppen soziale Kompetenz verlange, dass divergierende Netzkompetenzen aber auch zu Kommunikationsstörungen führen könnten. Die Darstellung in diesen drei Kapiteln entspricht über weite Strecken der Erstauflage des Buches, was insofern nicht überrascht, als hier grundlegende Begriffe der Sozialpsychologie erläutert und Phänomene diskutiert werden, die in den vergangenen Jahren keine grundsätzlichen Änderungen erfahren haben.

Das letzte Kapitel beginnt mit einer Zusammenfassung, in der Döring abermals für ihren medienökologischen Ansatz eintritt, der Soziales und Technisches, Mensch und Medienumwelt verbindet. Die Begründung ist überzeu- gend: „[S]oziale Prozesse sind die Basis für Technikentwicklung und umgekehrt strukturiert die Medientechnik die sozialen Austauschmöglichkeiten“ (S. 553). Abschließend lässt sie die im Buch behandelten sozialpsychologischen Aspekte der Internet-Forschung noch einmal Revue passieren und benennt die Desiderata sozialpsychologischer Forschung. Mit diesem Ausblick endet der Textteil des Buches, längst aber nicht das Buch selbst. Der Anhang umfasst über 100 Seiten. Er enthält ein Verzeichnis von gut recherchierten Internetadressen zur sozialwissenschaftlichen OnlineForschung (inkl. einschlägiger Zeitschriften) sowie eine umfangreiche, vollständig aktualisierte Literaturliste und einen Index, in dem alle wichtigen Stichwörter aufgeführt sind.

Aus den vorangehenden Ausführungen dürfte bereits deutlich geworden sein: Die „Sozialpsychologie des Internet" ist eine hervorragende Arbeit. Das Buch ist sachkundig geschrieben, es steht auf solider theoretischer und empirischer Basis und stellt nicht nur für Psychologen, sondern auch für Sprach-, Kommunikations- und Medienwissenschaftler eine wichtige Informationsquelle dar. Der Text ist sehr gut lesbar, angereichert mit Grafiken, Tabellen und Screenshots und aufgelockert durch zahlreiche Cartoons, die das Gesagte illustrieren und inhaltlich ergänzen. Einleitende Bemerkungen weisen auf den Inhalt der jeweiligen Kapitel hin, knappe Zusammenfassungen am Kapitelende fassen die zentralen Punkte zusammen, wichtige Begriffe sind kursiv gedruckt, zahlreiche internetspezifische Ausdrücke (z.B. Lurker, Nerd, Flasher u. a.) werden eingeführt und erläutert. Nicht zuletzt sei auch betont, dass der Text gründlich redigiert wurde. Auf den 662 Seiten finden sich nur wenige Fehler, das Layout ist ansprechend, die Seiten sind übersichtlich gestaltet. Und was die einleitend gestellte Fragen nach den Unterschieden zur Erstauflage betrifft, so kann abschließend festgehalten werden: Der Entwicklung des Internets sowie der Entwicklung der Internetforschung wird in der Neuauflage Rechnung getragen. Alle relevanten Zahlen und Internetadressen wurden aktualisiert, die jüngste Forschungsliteratur wurde aufgearbeitet, neue Fakten wurden in die Darstellung einbezogen. Kurzum: Auch die Neuauflage der „Sozialpsychologie des Internet“ ist eine anregende und informative Lektüre.

Christa Dürscheid 